\title{
On the Question of Ferromagnetism in Proton and He-Irradiated Carbon
}

\author{
J. Szczytko ${ }^{a}$, P. Juszyński ${ }^{a}$, L. Teliga $^{a}, \mathrm{~A} \mathrm{StonerT}^{b}$, \\ R. RATAJCZAK ${ }^{b}$, A. KORMAN ${ }^{b}$ AND A. TWARDOWSKI ${ }^{a}$ \\ ${ }^{a}$ Institute of Experimental Physics, Faculty of Physics, University of Warsaw \\ Hoża 69, 00-681 Warsaw, Poland \\ ${ }^{b}$ The Andrzej Sołtan Institute for Nuclear Studies \\ Hoża 69, 00-681 Warsaw, Poland
}

\begin{abstract}
We tried to repeat the observation of the ferromagnetic response in proton and He-irradiated carbon made by the group of Esquinazi et al. We used $\mathrm{He}^{+}$and $\mathrm{H}^{+}$beams focused on graphite sample. The amount of charge deposited in the sample was comparable to the amount of charge used by Esquinazi. Magnetic measurements were performed in SQUID magnetometer. The magnetization of the samples before and after irradiation was compared. We did not observe any ferromagnetic enhancement of magnetization of our irradiated samples. Even if experiment was not the same as Esqinazi's one, we can exclude some of the mechanisms of ferromagnetism proposed by Esquinazi.
\end{abstract}

PACS numbers: 75.20.Ck, 75.50.Dd

\section{Introduction}

Carbon-based materials are the promising candidates for future applications in novel electronics. Carbon nanotube transistors and logic gates, conducting carbon-based polymers have been already demonstrated. In addition, some forms of pure carbon should have magnetic properties, which in principle might be used in carbon-based spintronics. There are some theoretical predictions about carbon nano-stripes, schwarzite structures, H-doped graphite, in which electron spins on $\pi$-orbitals or broken $s p^{3}$-bonds have (in some cases) ferromagnetic alignment. The experiments seeking for ferromagnetism were performed but many of them have failed due to the presence of ferromagnetic impurities (mostly iron). Nevertheless, some of the experiments demonstrated ferromagnetism in pure carbon, particularly in carbon nano-foam $[1,2]$ and proton-irradiated highly oriented pyrolytic graphite (HOPG) [3-6]. In 2003 Esquinazi used a MeV proton beam and discovered that it trigged ferromagnetism of the HOPG sample. After irradiation the hysteresis 
loop was observed over the irradiated area using magnetic force microscopy and SQUID measurements.

Such magnetic system seems to be very interesting for both: basic science and possible application points of view. However, hardly any other experimental group has confirmed such experimental observation. This situation is embarrassing, because it is not clear if anybody really tried to verify the discoveries of Esquinazi et al. And since the lack of the phenomenon is difficult to be published in any paper (vide the rumor of "cold fusion" in 90-ties) there is no information about the efforts of other groups.

It was observed that samples of HOPG irradiated by protons became ferromagnetic [4-6]. The group of Esquinazi et al. used $2.25 \mathrm{MeV}$ proton beam and discovered that it caused ferromagnetic response from the sample. Purity of irradiated samples was checked by the Rutherford backscattering spectroscopy and particle induced X-ray emission spectra.

There are several theoretical attempts to explain this observation. Most of them take into account the existence of broken bonds and the presence of hydrogen. For instance Kusakube and Maruyama notes role of the mixed $s p^{2}$ and $s p^{3}$ hybridization in disordered graphite [7]. Lehtinen et al. investigated the role of carbon vacancies and hydrogen adatoms [8].

\section{Irradiation of the samples}

We have prepared four samples of pure (i.e. without detectable amount of iron) polycrystalline graphite (Table). The sample was irradiated with $2 \mathrm{MeV}$ protons from the 2 MV Van de Graaf accelerator at Soltan Institute for Nuclear

TABLE

The total amount of charge deposited in the samples.

\begin{tabular}{c|c|c|c|c|c}
\hline \hline & Used ions & $\begin{array}{c}\text { Energy } \\
{[\mathrm{MeV}]}\end{array}$ & $\begin{array}{c}\text { Charge } \\
{[\mathrm{mC}]}\end{array}$ & $\begin{array}{c}\text { Penetration } \\
\text { depth }[\mu \mathrm{m}]\end{array}$ & $\begin{array}{c}\text { Heating } \\
\text { atmosphere }\end{array}$ \\
\hline 1 & $\mathrm{H}^{+}$ & 2 & $>48$ & 5.2 & - \\
2 & $\mathrm{He}^{+}$ & 1 & $\approx 2$ & 3.5 & - \\
3 & $\mathrm{He}^{+}$ & 1 & $\approx 5$ & 3.5 & $\mathrm{NH}_{3}$ \\
4 & $\mathrm{He}^{+}$ & 2 & $\approx 7$ & 7.1 & $\mathrm{NH}_{3}$
\end{tabular}

Studies in Warsaw. Three samples were irradiated by $\mathrm{He}^{+}$for about $1 \mathrm{~h}$ and one by $\mathrm{H}^{+}$for over $21 \mathrm{~h}$. The diameter of the beam was about $5 \mathrm{~mm}$. The total amount of charge deposited in the samples is summarized in Table. The maximum dose obtained was as high as $0.22 \mathrm{C}$. Samples were mounted on a $\mathrm{Cu}$ holder with the carbon self-adhesive tape. Depending on the energy of the beam the penetration depth varied from 3.5 to $7.1 \mu \mathrm{m}$ from the surface (Fig. 1). In order to determine the role of the hydrogen, two samples were annealed in the ammonia $\mathrm{NH}_{3}$ atmosphere 


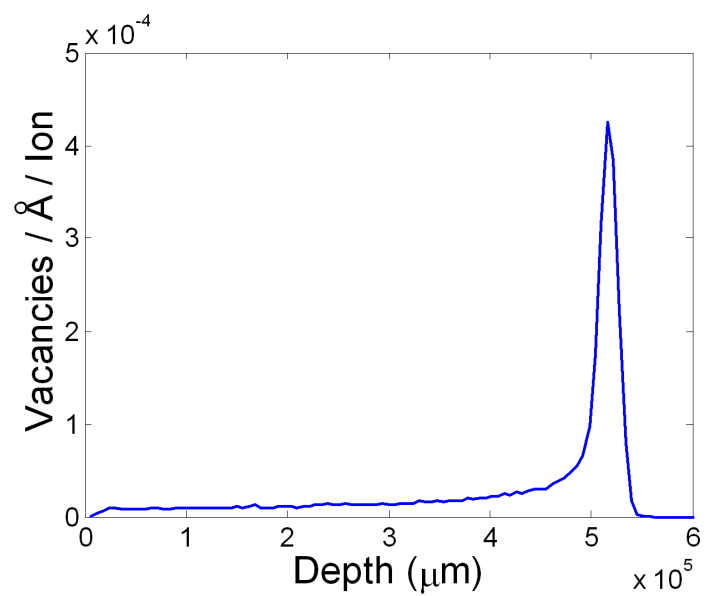

Fig. 1. Number of vacancies created per one carbon ion on $1 \AA$ depth inside the sample. The calculations for sample 1 (2 MeV proton beam).

at $500^{\circ} \mathrm{C}$. In this temperature ammonia decomposes into atomic hydrogen which can penetrate the graphite.

\section{Magnetization measurements}

The magnetic properties were measured using SQUID magnetometer. The sensitivity of our SQUID allows for the measurements as few as $10^{15}$ spins in the temperature range $2.0-300.0 \mathrm{~K}$ and magnetic field up to $6.0 \mathrm{~T}$.

\section{Results and discussion}

The amount of charge we used was comparable with the amount used by Esquinazi et al. The results of magnetization are shown in Fig. 2 together with the reference sample (non-irradiated). If any of the sample is magnetic one should see the deviation from the constant value of the magnetic susceptibility (magnetization divided by magnetic field). No such behavior was observed on any sample at any temperature.

Our experiment differs from the Esquinazi one. Firstly, the group of Esquinazi used mostly the micro-beam and our ion beam had several millimeters. However, in order to have comparable amount of vacancies we used long times of irradiation, so the concentration of vacancies is similar to the concentration achieved by Esquinazi. Secondly, we used polycrystalline sample, not HOPG. However, it should not have much influence on our result because the destruction of the lattice done by the ions is very local, as calculated by Kusakube and Maruyama [7] and Lehtinen et al. [8]. As long as we exclude the role of long distance order in sample the results should be similar in both cases. It seems that the only thing necessary to get the ferromagnetic response is presence of broken 


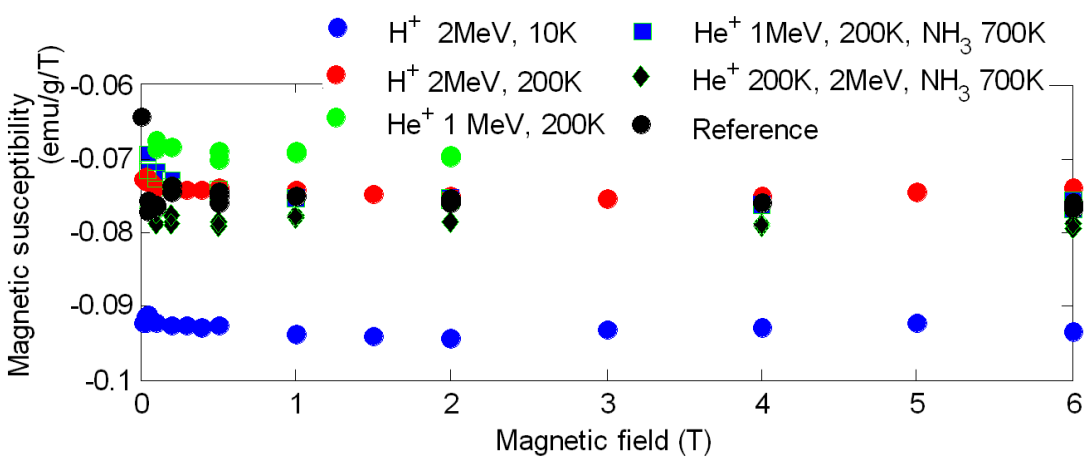

Fig. 2. The summary of magnetic measurements obtained on irradiated samples and the reference sample.

$\mathrm{C}-\mathrm{C}$ bonds, mixed $s p^{2}$ and $s p^{3}$ hybridization or hydrogen adatoms. However, we plan to repeat this experiment on HOPG samples as well to get the more detailed view on the situation.

\section{Summary}

In conclusion, we have tried to induce the ferromagnetism in polycrystalline graphite using different irradiation of $\mathrm{H}^{+}$and $\mathrm{He}^{+}$ions combined with the doping of hydrogen. We did not observe any sight of enhanced magnetization of all irradiated samples. The further investigations on HOPG samples will be conducted.

\section{Acknowledgments}

The partial support from European Network of Excellence MTKD-CT-2005029671 is acknowledged.

\section{References}

[1] A.V. Rode, E.G. Gamaly, A.G. Christy, J.G. Fitz Gerald, S.T. Hyde, R.G. Elliman, B. Luther-Davies,A. I. Veinger, J. Androulakis, J. Giapintzakis, Phys. Rev. $B$ 70, 054407 (2004).

[2] A.V. Rode, E.G. Gamaly, A.G. Christy, J. Fitz Gerald, S.T. Hyde, R.G. Elliman, B. Luther-Davies, A.I. Veinger, J. Androulakis, J. Giapintzakis, J. Magn. Magn. Mater. 290, 298 (2005).

[3] R. Höhne, P. Esquinazi, Adv. Mater. 14, 753 (2002).

[4] P. Esquinazi, D. Spemann, R. Höhne, Phys. Rev. Lett. 91, 227201 (2003).

[5] S. Talapatra, P.G. Ganesan, T. Kim, R. Vajtai, M. Huang, M. Shima, G. Ramanath, D. Srivastava, S.C. Deevi, P.M. Ajayan, Phys. Rev. Lett. 95, 097201 (2005).

[6] P. Esquinazi, R. Höhne, K.-H. Han, A. Setzer, D. Spemann, T. Butz, Carbon 42, 1213 (2004).

[7] K. Kusakabe, M. Maruyama, Phys. Rev. B 67, 092406 (2003).

[8] P.O. Lehtinen, A.S. Foster, Yuchen Ma, A.V. Krasheninnikov, R.M. Nieminen, Phys. Rev. Lett. 93, 187202 (2004). 